\title{
Bias Image Correction Via Stationarity Maximization
}

\author{
T. Dorval, A. Ogier, and A. Genovesio \\ Image Mining Group \\ Institut Pasteur Korea \\ 39-1, Hawolgok-dong, Seongbuk-gu, Seoul, 136-791, Korea*
}

\begin{abstract}
Automated acquisitions in microscopy may come along with strong illumination artifacts due to poor physical imaging conditions. Such artifacts obviously have direct consequences on the efficiency of an image analysis algorithm and on the quantitative measures. In this paper, we propose a method to correct illumination artifacts on biological images. This correction is based on orthogonal polynomial modeling, combined with stationary maximization criteria. To validate the proposed method we show that we improve particle detection algorithm.
\end{abstract}

Index Terms: Biomedical Image Processing, Image Analysis, Image Enhancement, Object Detection, Biomedical Microscopy.

\section{Introduction}

Modern microscopy and robotic technologies allow a very large amount of visual information to be collected and analyzed automatically. These new systems make the visual inspection of the pictures totally obsolete, but also give a chance for an objective quantitative measurements on cell experiments. Nuclei, endosomes and other particles detection is a common request for biological image analysis. However illumination artifacts systematically occur on 2D cross-section confocal microscopy imaging platforms. These biases can strongly corrupt a higher level image analysis such as segmentation, fluorescence evaluation or even pattern extraction / recognition 123. To overcome this drawback many methods have already been proposed in literature. A reader can refer to [4 for a comparative evaluation of the most common intensity inhomogeneities correction techniques. In this paper, we make the assumption that bias generates a non stationary process which can be corrected by orthogonal polynomial modeling. This paper presents a new fully automated bias correction methodology, which improve low level biological image analysis such as segmentation or particle detection algorithm. A relevant protocol validates the correction algorithm and shows outperforming extraction on corrupted images. This paper deals with $2 \mathrm{D}$ fluorescence confocal microscopy, but the framework can be easily extended to

\footnotetext{
* The authors thank T. Christophe, R. Grailhe and P. Sommer from Institut Pasteur Korea for providing valuable confocal microscopic images.
} 
many other biological imaging techniques. In section 2 the stationarity definition is recall. In section 3 we describe the polynomials correction framework. In section 4 we propose to validate this methodology by classical features detection in cell images. Finally, we conclude in section 5.

\section{Stationarity Definitions}

In signal processing, according to the traditional definition, a time series, $X_{t}$, is stationary in the wide sense, if:

$$
\left\{\begin{array}{l}
\forall t \in \mathbb{Z}, E\left(\left|X_{t}^{2}\right|\right)<\infty, \\
\forall t \in \mathbb{Z}, E\left(X_{t}\right)=m, \\
\forall(t, h) \in \mathbb{Z}^{2}, \operatorname{Cov}\left(X_{t}, X_{t+h}\right)=E\left[\left(X_{t+h}-m\right)\left(X_{t}-m\right)\right]=\gamma(h),
\end{array}\right.
$$

in which $E($.$) is the expectation value and \operatorname{Cov}($.$) is the covariance function.$

If we refer to the terminologies from Nelson and Plosser [5, two classes of non stationary process exist: the Trend Stationary (TS) process and the Differency Stationary (DS) process of ordre $d$. For TS process, the non stationarity follows a determinism model and can be written:

$$
X_{t}=g(t)+\epsilon_{t},
$$

with $g(t)$ a time dependent function and $\epsilon_{t}$ a stationary stochastic process. A simple example of a TS process is a linear trend disturbed by a white noise. In this case $g(t)=a_{0}+a_{1} t$ with $a_{0}, a_{1} \in \mathbb{R}$ and $\epsilon_{t}$ i.i.d $\mathcal{N}\left(0, \sigma^{2}\right)$.

The DS process is given by $X_{t}=(1-L)^{d} X_{t}$, with $\mathrm{L}$ the lag operator $\left(L X_{t}=\right.$ $\left.X_{t-1} \forall t \in \mathbb{Z}\right)$ and $d \in \mathbb{Z}$. Thus, for a DS process the trend is not a determinism one but stochastic. A famous example is the pure random walk defined by $X_{t}=$ $X_{t-1}+\epsilon_{t}$.

Shading phenomenon is often defined as a smooth intensity variation, leading to a nonuniform illumination of the image. Based on this definition, we assume that the corrupted images can be seen as a TS process. Moreover, we consider that $g(t)$ can be modelled by a polynomial $P(t)$. As we are dealing with $2 \mathrm{D}$ images, the time $t$ is replaced by the spatial coordinates $(x, y)$. Thus, for the following, $P(t)$ and $\epsilon_{t}$ will be respectively noted $P(x, y)$ and $\epsilon_{x, y}$ where $(x, y)$ is the spatial location of a specific point within the image.

\section{Bias Image Correction Via Stationarity Maximization}

\subsection{Legendre Polynomials Approximation}

In our context, we consider that each pixel $f(x, y)$ of an image $f$ is a combination of its real intensity $u(x, y)$, an illumination bias artifact $b(x, y)$, and an additive white Gaussian noise $\epsilon_{x, y} \equiv \mathcal{N}\left(0, \sigma_{\text {noise }}^{2}\right)[6$. The relation is given by:

$$
f=u b+\epsilon .
$$


According to (2), to correct each picture, we divide the observed signal $f$ by the estimated bias $\tilde{b}_{m, n}$. Thus, this equation becomes:

$$
\frac{f}{\tilde{b}_{m, n}}=\frac{u b}{\tilde{b}_{m, n}}+\frac{\epsilon}{\tilde{b}_{m, n}}
$$

where $(m, n)$ are respectively the $x$ and $y$ polynomial orders. We systematically apply a Gaussian filtering to the image prior to estimating the bias. Hence, $\epsilon<<\tilde{b}_{m, n}$ can be omitted and we obtain:

$$
\frac{f}{\tilde{b}_{m, n}} \approx \frac{u b}{\tilde{b}_{m, n}} \approx u
$$

To perform this correction, we model the trend $\tilde{b}_{m, n}$ of the intensity distribution by using orthogonal polynomial functions [7].

The orthogonal polynomials $p .(x)$ is computed according to the following recurrence relation [8]:

$$
\left\{\begin{array}{l}
p_{0}(x)=1 \\
p_{1}(x)=x \\
p_{m+1}(x)=\left(a_{m}+x b_{m}\right) p_{m}-c_{m} p_{m-1},
\end{array}\right.
$$

where the triplet $\left(a_{m} ; b_{m} ; c_{m}\right)$ defines a specific polynomial family. In addition to its orthogonality properties, the Legendre polynomial is particularly well suited in our case because of its constant density and its limited interval $(x \in[-1 ;+1])$. A set of $2 \mathrm{D}$ orthogonal polynomials basis can be computed by a linear combination of 1D Legendre polynomials. Thus, polynomial images $P_{m, n}(x, y ; \mathbf{A})$ $\left((m, n) \in \mathbb{N}^{+}\right)$are computed given the following formula:

$$
P_{m, n}(x, y ; \mathbf{A})=\frac{1}{(n+1)(m+1)} \sum_{j=0}^{n} \sum_{i=0}^{m} \alpha_{i, j} p_{i}(x) p_{j}(y),
$$

where $\alpha_{i, j} \in \mathbb{R}$ and $(x, y) \in[-1,+1]$ and $\mathbf{A}$ is a matrix containing the $\alpha_{i, j}$ values. Figure 1 shows the 2D Legendre polynomial basis from degree 0 to degree 3.

For each corrupted image $f$, the evaluation of the $(n+1)(m+1)$ parameters $\alpha_{i, j}$ of (3) is based on a least-square minimization of the functional $E_{m, n}(\mathbf{A})$ given by:

$$
E_{m, n}(\mathbf{A})=\left(\sum_{y} \sum_{x}\left(P_{m, n}(x, y ; \mathbf{A})-f(x, y)\right)^{2}\right)^{\frac{1}{2}},
$$

using the multi-dimensional Polak-Ribiere conjugate gradient minimization method 9]. The minimization result $\min \left(E_{m, n}(\mathbf{A})\right)$ gives us the $\alpha_{i, j}$ values corresponding to the estimated bias $\tilde{b}_{m, n}$ (see figures 2 \& 3 ) for a specific $(m, n$ ) combination. 


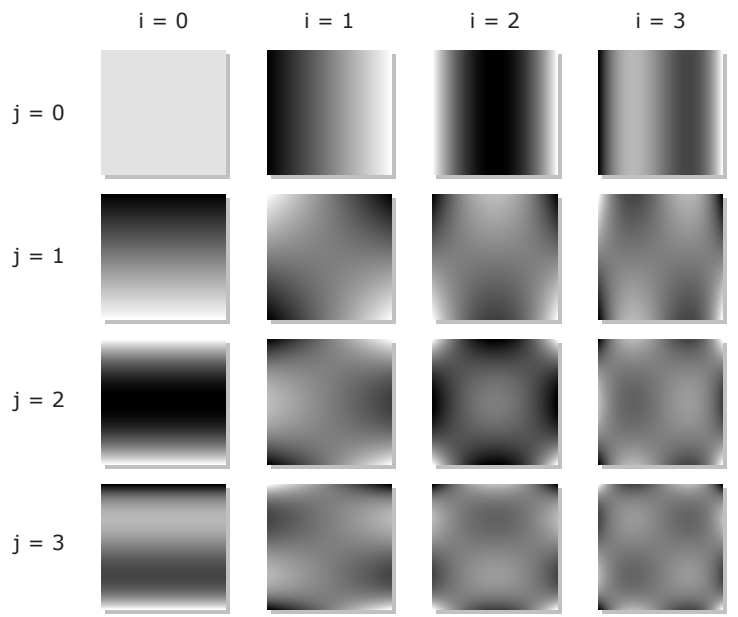

Fig. 1. 2D Legendre polynomials basis for low degree polynomials

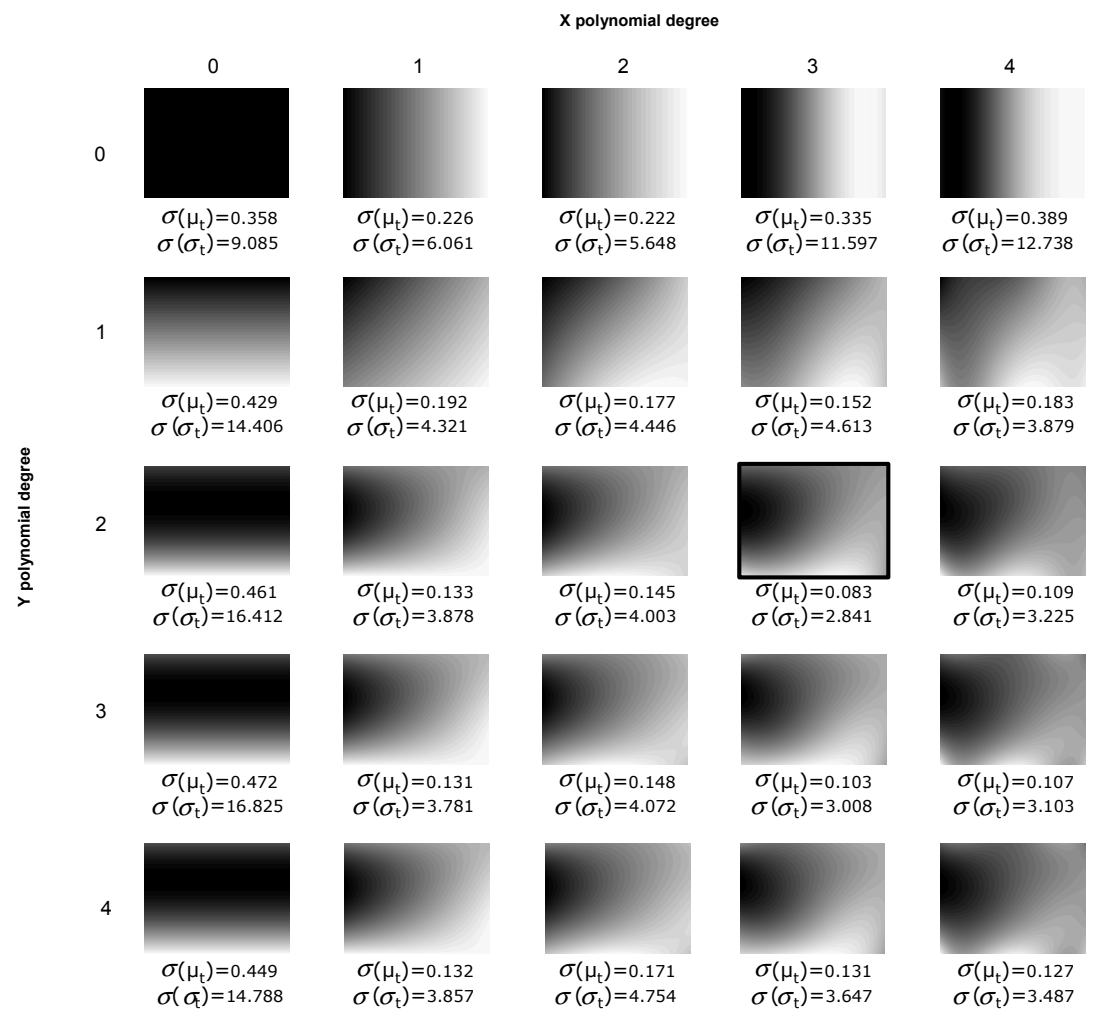

Fig. 2. Bias estimations with $(m, n) \in[0 ; 4]$ corresponding to $I_{1}$ in figure 3 The outlined bias picture $(m=3 ; n=2)$ corresponds to the optimal correction map according to the stationarity criterion. 


\subsection{Stationarity Maximization}

Obviously, the best correction results (i.e. satisfying as well as possible the stationarity assumptions) are not given by the highest values of the $(m, n)$ couple. Indeed, with a too high polynomial degree, the correction map will not only fit the trend of the illumination artifact but the image details also, which may not be relevant for the correction. Experiments corroborate this assumption and lead us to determine these degrees in an automated way. For this, we extract this optimal result within the optimizations realized for $(m, n) \in[0 ; 4]$ and defined in (4).

The first one imposes that the energy of the signal is finite. This property is always verified due to the discretization phenomenon.

The second one, the most important in the case of a trend stationary process, imposes that the expectation is not related to the temporal (or spatial) variable. To evaluate the spatial variation of the local mean $\mu_{t}$ over the image, we compute the standard deviation $\sigma\left(\mu_{t}\right)$ of this signal on a sliding window. The size of the window must be large enough to provide a significant statistical representation of the imaged phenomena.

Removing the trend in a TS process can introduce an unitary root (i.e a correlation between the variable in time $(t)$ and in $\left.(t-h), h \in \mathbb{N}^{+}\right)$and thus turns a TS into a DS process. In order to discriminates these two cases, we verify the independence of the second order moment $\sigma^{2}$ over the time, satisfying the third property of (11) for $h=0$.

Biased Images: $f$

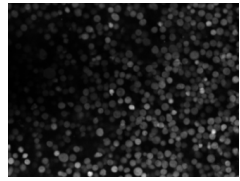

$\sigma\left(\mu_{t}\right)=0.358$

$\sigma\left(\sigma_{\mathrm{t}}\right)=9.085$

Estimated Bias: b

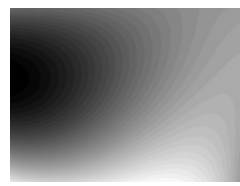

$b_{2,3}$

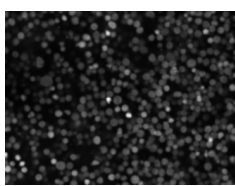

$\sigma\left(\mu_{\mathrm{t}}\right)=0.083$

$\sigma\left(\sigma_{\mathrm{t}}\right)=2.841$

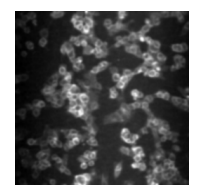

$\sigma\left(\mu_{\mathrm{t}}\right)=0.249$

$\sigma\left(\sigma_{\mathrm{t}}\right)=7.583$

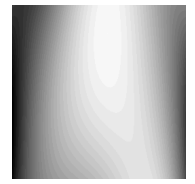

$b_{3,2}$

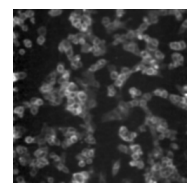

$\sigma\left(\mu_{\mathrm{t}}\right)=0.157$ $\sigma\left(\sigma_{\mathrm{t}}\right)=4.791$

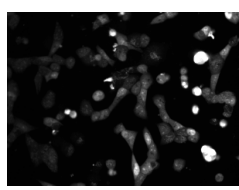

$\sigma\left(\mu_{\mathrm{t}}\right)=0.349$ $\sigma\left(\sigma_{\mathrm{t}}\right)=28.056$

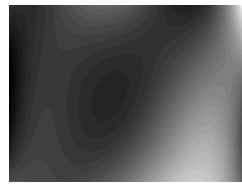

$\mathbf{b}_{4,2}$

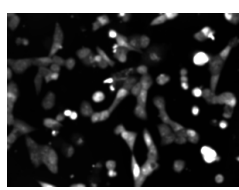

$\sigma\left(\mu_{\mathrm{t}}\right)=0.173$

$\sigma\left(\sigma_{\mathrm{t}}\right)=17.09$

Fig. 3. Examples of biased images obtained by three different confocal microscopes for three different biological applications. The optimal correction maps are not given by the maximal order polynomials, but really depend of the illumination artifacts trend. 
Finally, the optimal result corresponds to the estimated bias that minimize the variation of this two moments. Figure 2 displays a set of bias estimations corresponding to the image $I_{1}$ in figure 3. The outlined bias picture gives the best result according to our stationary criteria. Figure 3 displays three different biased images and their optimal correction maps associated. We can noticed that the maximal stationarity is reached for various orthogonal polynomial degrees demonstrating the accuracy of our primary assumption.

\section{Biological Applications and Results}

This section describes a framework for extracting circular objects within cells in a relevant way. This method can be applied to any kind of spot detection requirement, such as endosomes localization. In our purpose, objects can be considered rotationally invariant. Thus, the Hessian $\mathcal{H}_{\sigma}$ operator is perfectly appropriate, where the parameter $\sigma$ is selected to match the spot candidate size. Thanks to this value, we are able to extract a wide variety of biological objects. $\mathcal{H}_{\sigma}$ allows us to define two curvature maps $C_{M}$ and $C_{G}$ coming respectively from the mean and the Gaussian curvatures as defined in 10. To discriminate the "dome" topographic class from the other classes, we keep only the positive values of $C_{M}$ and $C_{G}$.

The curvature map $u_{c u r v}$ is computed by $u_{c u r v}=C_{M} \cdot C_{G}$ (see figure 48). As it is usual in cytometry imaging, one wavelength is dedicated to nucleus and/or cytoplasm imaging. This channel allows us to create a binary cells mask $u_{\text {mask }}$ using a simple segmentation method such as K-means clustering (see figure 4d). It is important to notice that the robustness of this step is highly correlated to the restoration step quality.

Pixels are weighted by an approximation of the Euclidean distance obtained by computing a connexity distance field. The distance map $u_{\text {dist }}$ obtained is combined to $u_{\text {curv }}$ by $u_{\text {res }}=u_{\text {dist }} . u_{\text {curv }}$ (see figure 4 $\mathrm{g}$ ). A threshold $\tau_{\text {min }}$ is then applied to avoid false detections within $u_{\text {res }}$.

To conclude the process, a local maxima extraction is done. In our specific application, the minimum distance allowed between two successive extracted spots is given by $3 \sigma$.

To assess the robustness of our correction method, we have created a ground truth based on three different biological assays. The ground truth was built

Table 1. $\kappa$ index and overlap coefficient for three different experiments. $\kappa$ and $C_{\text {over }}$ values higher than 0.7 usually represent very good results.

\begin{tabular}{|l||c|c||c|c|}
\hline & $\kappa(f)$ & $C_{\text {over }}(f)$ & $\kappa(u)$ & $C_{\text {over }}(u)$ \\
\hline experiment 1 & 0.683 & 0.531 & 0.898 & 0.814 \\
\hline experiment 2 & 0.636 & 0.467 & 0.836 & 0.747 \\
\hline experiment 3 & 0.542 & 0.421 & 0.867 & 0.813 \\
\hline
\end{tabular}


by manually selecting the right objects corresponding to the expected feature locations. Then to qualify these results, two statistical coefficients are computed:

- the kappa index $(\kappa)$ defined by [1] :

$$
\kappa=2 \frac{\#(g t \cap d)}{\#(d)+\#(g t)} ;
$$

- the overlapping coefficient defined by:

$$
C_{\text {over }}=\frac{\#(g t \cap d)}{\#(g t \cap d)+\#(f p)+\#(f n)},
$$

where $g t$ is the ground truth, $d$ the detected objects, $f p$ and $f n$ the false positives and false negatives respectively, and \# the cardinal of the set. Table 1 shows the results and underlines the accuracy of the correction process for different experiments.

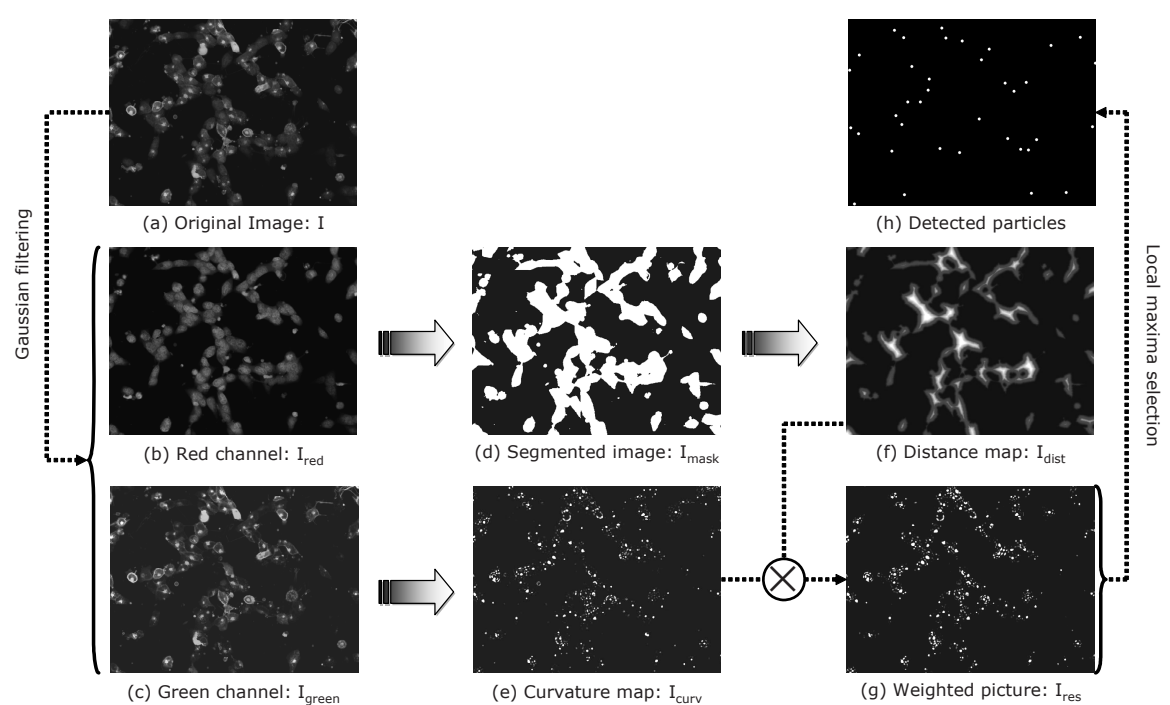

Fig. 4. Step by step particles detection in 2-channel images. (a) original image. (h) detected particles locations.

\section{Conclusion}

In this paper, we have shown that illumination bias can have important consequences on low level biological image processing quality. To overcome this drawback, we presented a novel shading correction approach based on the image stationary maximization via Legendre polynomials modeling. This image enhancement was used for correcting different cell images. The visual quality of 
the corrected results is high and was confirmed by a ground truth based features detection. As, this method produced significant improvement of traditional biological objects detection under different imaging conditions, it could be used as a pre-processing step for any kind of higher level process. In the future, we plan to propose a $3 \mathrm{D}$ implementation of this shading correction framework.

\section{References}

1. Jones, T.R., Carpenter, E., Sabatini, D.M., Golland, P.: Methods for high-content, high-throughput image-based cell screening. In: MIAAB 2006 Workshop Proceedings (2006)

2. Dorval, T., Ogier, A., Dusch, E., Emans, N., Genovesio, A.: Bias free features detection for high content screening. In: 4th IEEE International Symposium on Biomedical Imaging, Metro Washington, DC, USA (2007)

3. Ogier, A., Dorval, T., Genovesio, A.: Biased image correction based on empirical mode decomposition. In: IEEE International Conference on Image Processing 2007 (ICIP 2007), San Antonio, Texas, USA (2007)

4. Tomazevic, D., Likar, B., Pernus, F.: Comparative evaluation of retrospective shading correction methods. Journal of Microscopy 208(3), 212-223 (2002)

5. Nelson, C., Plosser, C.: Trends and random walks in macroeconomic time series: Some evidence and implications. Journal of monetary economics 10, 139-162 (1982)

6. Styner, M., Brechbuhler, C., Szekely, G., Gerig, G.: Parametric estimate if intensity inhomogeneities applied to mri. IEEE Transactions on Medical Imaging 19, 153165 (2000)

7. Fee, G.: Linear least-squares data fitting with orthogonal polynomials. In: Maple Summer Workshop (2002)

8. Abramowitz, M., Stegun, I.A.: Handbook of Mathematical Functions with Formulas, Graphs, and Mathematical Tables. ninth dover printing, tenth gpo printing edn. Dover, New York (1970)

9. Polak, E.: 2.3. In: Computational Methods in Optimization, Academic Press, New York (1971)

10. Wilson, R.C., Hancock, E.: Consistent topographic surface labelling. Pattern Recognition Letters 32, 1211-1223 (1999)

11. Cohen, J.: A coefficient of agreement for nominal scales. Educational and Psychological Measurements 20(3), 27-46 (1960) 